\title{
(Don't) panic in the scanner! How panic patients with agoraphobia experience a functional magnetic resonance imaging session
}

\author{
Ulrike Lueken $^{\text {a,b, }}{ }^{*}$, Markus Muehlhan ${ }^{\text {a,b }}$, Hans-Ulrich Wittchen ${ }^{\text {a }}$, Thilo Kellermann ${ }^{\text {c,d }}$, \\ Isabelle Reinhardt ${ }^{c, d}$, Carsten Konrad ${ }^{\text {e,f }}$, Thomas Lang ${ }^{\mathrm{g}, \mathrm{h}}$, André Wittmann ${ }^{\mathrm{i}}$, Andreas \\ Ströhle $^{\mathrm{i}}$, Alexander L. Gerlach ${ }^{\mathrm{j}, \mathrm{k}}$, Adrianna Ewert ${ }^{1}$, Tilo Kircher ${ }^{\mathrm{e}}$
}

\author{
a Institute of Clinical Psychology and Psychotherapy, Department of Psychology, Technische Universität \\ Dresden, Dresden, Germany \\ b Neuroimaging Center, Department of Psychology, Technische Universität Dresden, Dresden, Germany \\ c Department of Psychiatry and Psychotherapy, RWTH Aachen University, Aachen, Germany \\ d Jülich Aachen Research Alliance (JARA), Translational Brain Medicine, Aachen, Germany \\ e Department of Psychiatry and Psychotherapy, Philipps-University Marburg, Marburg, Germany \\ f Department of Psychiatry, University of Münster, Münster, Germany \\ g Centre for Clinical Psychology and Rehabilitation, University Bremen, Bremen, Germany \\ h Christoph-Dornier-Foundation for Clinical Psychology, Bremen, Germany \\ i Department of Psychiatry and Psychotherapy, Charité Campus Mitte, Charité, Universitätsmedizin Berlin, \\ Berlin, Germany \\ j Institute of Clinical Psychology and Psychotherapy, University of Münster, Münster, Germany \\ $\mathrm{k}$ Institute of Clinical Psychology and Psychotherapy, Department of Psychology, University of Cologne, \\ Cologne, Germany \\ 1 Department of Clinical Radiology, University Hospital Münster, Münster, Germany
}

\begin{abstract}
Although functional magnetic resonance imaging (fMRI) has gained increasing importance in investigating neural substrates of anxiety disorders, less is known about the stress eliciting properties of the scanner environment itself. The aim of the study was to investigate feasibility, self-reported distress and anxiety management strategies during an fMRI experiment in a comprehensive sample of patients with panic disorder and agoraphobia (PD/AG). Within the national research network PANIC-NET, $n=89$ patients and $n=90$ controls participated in a multicenter fMRI study. Subjects completed a retrospective questionnaire on self-reported distress, including a habituation profile and exploratory questions about helpful strategies. Drop-out rates and fMRI quality parameters were employed as markers of study feasibility. Different anxiety measures were used to identify patients particularly vulnerable to increased scanner anxiety and impaired data quality. Three $(3.5 \%)$ patients terminated the session prematurely. While drop-out rates were comparable for patients and controls, data quality was moderately impaired in patients. Distress was significantly elevated in patients compared to controls; claustrophobic anxiety was furthermore associated with pronounced distress and lower fMRI data quality in patients. Patients reported helpful strategies, including motivational factors and cognitive coping strategies. The feasibility of large-scale fMRI studies on PD/AG patients could be proved. Study designs should nevertheless acknowledge that the MRI setting may enhance stress reactions. Future studies are needed to investigate the relationship between self-reported distress and fMRI data in patient groups that are subject to neuroimaging research.
\end{abstract}

Keywords: fMRI; Neuroimaging; Anxiety disorders; Panic disorder; Agoraphobia; Stress 


\section{Introduction}

Although functional magnetic resonance imaging (fMRI) has gained increasing importance in investigating neural substrates of anxiety disorders (Paulus, 2008), less is known about the stress eliciting properties of the scanner environment itself. Reports about routine care patients indicate that an MRI examination can produce subjective distress (Dantendorfer et al., 1997; Flaherty and Hoskinson, 1989; Kilborn and Labbe, 1990; Mackenzie et al., 1995; McIsaac et al., 1998) or trigger long-term claustrophobia (Fishbain et al., 1988; Kilborn and Labbe, 1990) and panic attacks (Spiegelhalder et al., 2009) in subjects with no previous psychiatric history. Pre-existing claustrophobia may moreover predict distress and panic symptoms in the scanner (McIsaac et al., 1998). Adverse reactions not only constitute a significant problem for patients: they can affect the performance of (f)MRI studies by increasing the number of declined appointments, premature terminations of scans, or impair data quality (Dantendorfer et al., 1997).

Scanner distress ranges from moderate apprehension (approx. 30\%), to severe panic and claustrophobia experienced by $5-10 \%$ of routine care patients (Melendez and McCrank, 1993). Among anxiety patients, those suffering from panic disorder and agoraphobia (PD/AG) are particularly vulnerable to the stress eliciting properties of the MRI, which is a highly agoraphobic/claustrophobic stimulus. Comprehensive assessments of scanner distress in PD are still lacking, but Bystritsky et al. (2001) found elevated panic symptoms in patients compared to controls. Maddock et al. (2003) stated that four out of six patients reported feeling anxious throughout the scan. Pillay et al. (2006) observed that although all eight patients were able to complete the scan, they showed initial signs of hesitation. Interpretation of these observations is however limited by the small number of patients and lack of quantitative assessment tools.

The aim of the present study was to investigate scanner-related effects on self-reported distress and data quality in a multicenter fMRI study on $n=89 \mathrm{PD} / \mathrm{AG}$ patients, addressing the following research questions:

1) Are large-scale fMRI studies in PD/AG patients (dropout-rates, data quality) feasible?

2) Do patients and controls differ in their amount of distress before, during and after the session?

3) Can we identify vulnerable patients using different measures of anxiety?

4) Management strategies: what can we do to make patients feel more comfortable?

\section{Experimental procedures}

\subsection{Multicenter study PANIC NET}

The fMRI study was embedded within a national research network (PANIC-NET; Arolt et al., 2009; Gloster et al., 2009) that is characterized by a multilevel and multicenter research structure, encompassing a randomized controlled clinical trial on cognitive behavioural therapy (CBT) and experimental add-on studies on fear circuit mechanisms in PD/AG. Eight centers participated in the clinical trial (Aachen, Berlin-Adlershof, Berlin-Charité, Bremen, Dresden, Greifswald, Münster, and Würzburg) treating n= 369 patients who met DSM-IV 
criteria for PD/AG. Four centers (Aachen, Berlin-Charité, Dresden, and Münster) also participated in the fMRI study which had been approved by all responsible ethics committees of the participating centers (research cluster P7: "Neuronal plasticity following cognitivebehavioral therapy in patients with panic disorder: a multicenter 3-Tesla study using fMRI").

\subsection{Subjects}

Eighty-nine PD/AG patients and 90 healthy controls participated in the fMRI study; $n=87$ patients also participated in the clinical trial. Patient inclusion criteria encompassed a primary diagnosis of PD/AG according to the criteria of DSM-IVTR as assessed by the Composite International Diagnostic Interview (CAPI-WHO-CIDI; DIAX-CIDI version; Wittchen and Pfister, 1997), a score $\geq 18$ at the Hamilton-Anxiety-Scale (SIGH-A; Shear et al., 2001), a score $\geq 4$ at the Clinical Global Impressions Scale (CGI; Guy, 1976), age between 18 and 60 years. Exclusion criteria encompassed psychotic or bipolar I disorders, current threshold alcohol or substance dependence, current suicidal tendency, borderline personality disorder, organic mental disorders, concurrent psychotherapeutic/ psychopharmacological treatments, severe cardiovascular, renal, and neurological diseases, colour blindness and any MRI-related exclusion criteria. Exclusion criteria were either assessed by entry into the clinical trial or prior to participation in the fMRI study. The control group was matched for gender, education, age, and handedness. Subjects were excluded in case of any current or lifetime diagnosis of any mental disorder (except misuse of alcohol and nicotine dependence), neurological disorders, central-nervous medication within the last four weeks, colour blindness, and MRI-related contraindications.

\subsection{Procedure}

Following inclusion into the clinical trial, patients were asked for participation in the fMRI study. Controls were recruited via advertisements and flyers. Subjects were scheduled an appointment (baseline assessment) at the scanner to obtain informed consent and to offer a try-out (e.g. lying in the tomograph). The fMRI session consisted of three functional runs and a structural scan (overall duration: $60 \mathrm{~min}$ ), after which patients completed a questionnaire on subjective experiences during the fMRI session. All subjects were invited for a second fMRI assessment (post assessment) after completion of the therapy; only data from the baseline assessment are reported here. All subjects received $25 €$ for each assessment.

\subsection{Measurements}

\subsubsection{Anxiety measures and scanner-related distress}

Within two weeks of the fMRI appointment, a psychometric characterization of patients was obtained by the clinical trial (see Gloster et al., 2009). The following measures were selected for present analyses: SIGH-A, Panic and Agoraphobia Scale (PAS; Bandelow, 1999), Anxiety Sensitivity Index (ASI; Reiss et al., 1986), Claustrophobia Questionnaire (CLQ; Radomsky et al., 2001), and Beck Depression Inventory (BDI II; Beck et al., 1996).

The fMRI questionnaire was completed directly after the session, assessing a) degree of exhaustion caused by the scanner session, b) duration of anxiety, c) overall maximum anxiety, and d) a habituation profile on anticipatory anxiety before scanning (pre-start anxiety), at beginning (start-scan anxiety) and after completion (post-scan anxiety). All ratings were scored on a 9-point Likert scale. Patients were furthermore asked what had helped them to 
participate in the fMRI study. Questionnaire data are available for $n=76$ patients and $n=55$ controls.

\subsection{2. fMRI paradigms and quality parameters}

The paradigms addressed different functions of the fear circuit (see Arolt et al., 2009), encompassing fear conditioning, interoceptive processing and symptom provocation using agoraphobic visual stimuli. MRI images were acquired on 3-Tesla MRI scanners. Standard head coils and headphones were applied. Visual stimuli were presented using video goggles (Aachen, Dresden: VisuaStim Digital, Northridge, California) or beamer projection (Münster, Berlin). Functional measurements were obtained using T2* weighted gradient echo planar images (EPI) sequences.

The quality of fMRI time-series is crucial for within- and between-site reliability. Realignment parameters (SPM5; http://www.fil.ion.ucl.ac.uk/spm/) were used to quantify the amount of motion artifacts. We furthermore used the automated quality assurance routine introduced by Stocker et al. (2005), which gives one dispersion measure per volume (averaged across voxels) expressed as percent signal change relative to the mean of the timeseries (e.g., percent signal fluctuation). The measure is based on temporal fluctuations within a time-series which is expressed by the difference of each scan (e.g. time-point) to the mean image of the series. Higher average differences indicate relatively higher temporal variation in the signal. The dispersion measure is based on realigned data (Stocker et al., 2005).

Realignment parameters (averaging $\mathrm{x}, \mathrm{y}$, and $\mathrm{z}$ values) and dispersion measures were calculated for each paradigm in order to analyze the time course of data quality within the session. In addition, mean translation and rotation indicators and dispersion measures were averaged to characterize overall quality of fMRI data in patients and controls.

\subsection{Data analysis}

Sample characteristics and drop-out rates were tested using t-tests for independent samples, chi $^{2}$ or Fisher's exact tests. Since fMRI quality parameters were skewed distributed, Friedman tests and Mann-Whitney-U-tests were used to test for changes within the session from the first to the third paradigm (separately for patients and controls) and for group differences (patients (stratified for CLQ values) vs. controls) in overall data quality. Gender effects on fMRI quality parameters and subjective distress were analyzed using Mann-Whitney-U-tests and t-tests, respectively. Differences in self-reported distress between patients and controls were analyzed using t-tests (degree of exhaustion, duration of anxiety, and maximum anxiety), and a two-way analysis of variance (ANOVA; within-subjects-factor "time course", between-subjects factor "group"). Associations between anxiety measures and self-reported distress were evaluated using Pearson's correlations. When testing differences in distress between high vs. low claustrophobic patients and controls the BDI II score was entered as a covariate into the ANCOVA model in order to control for unspecific depressive symptoms. Greenhouse-Geisser adjustments were applied when appropriate. Effect sizes (Cohen, 1983) were computed for all analyses on hypotheses (Cohen's $d \geq 0.20, r \geq 0.10$ and $f^{2} \geq 0.10$ : small effects; $d \geq 0.50, r \geq 0.30$ and $f 2 \geq 0.25$ : medium effects; $d \geq 0.80, r \geq 0.50$ and $f^{2} \geq 0.40$ : large effects). A level of $\alpha<0.05$ indicated statistical significance. All analyses were carried out using PASW 17.0. 


\section{Results}

\subsection{Sample characteristics, dropout-rates and data quality}

Sample characteristics are displayed in Table 1, separately for the entire sample and the sample for which the fMRI questionnaire was available. The majority of patients $(n=61$; $68.54 \%)$ and controls $(n=61 ; 67.78 \%)$ were female $\left(\mathrm{chi}^{2}(1)=0.012, \mathrm{p}=0.913\right)$; mean age (SD) averaged at 36.48 years (11.31) for patients and 35.56 years $(10.87)$ for controls $(t$ $(176)=0.56, p=0.580)$. Patients' clinical characteristics indicated moderate to severe severity for general and symptom-specific anxiety as measured by SIGH-A, PAS, ASI, and CLQ. In order to assure that the reduced sample for which questionnaire data were available was representative for the entire sample, differences in demographic, clinical, and data quality parameters were tested. Both patient and control samples were comparable in all variables (patients sample 1 vs. 2 : gender: $\operatorname{chi}^{2}(1)=0.24, \mathrm{p}=0.62$; age: $\mathrm{t}(163)=0.44, \mathrm{p}=0.66$; SIGH-A: $\mathrm{t}$ $(161)=-0.42, \mathrm{p}=0.67$; PAS: $\mathrm{t}(161)=-0.69, \mathrm{p}=0.489$; ASI: $\mathrm{t}(161)=-0.51, \mathrm{p}=0.613$; CLQ: $\mathrm{t}$ $(161)=-0.79, p=0.433$; BDI II: $\mathrm{t}(161)=-0.48, \mathrm{p}=0.634$; dispersion measure: $\mathrm{Z}=-0.89$, $\mathrm{p}=0.373$; translation: $\mathrm{Z}=-0.38, \mathrm{p}=0.707$; rotation: $\mathrm{Z}=-0.14, \mathrm{p}=0.890$; controls sample 1 vs. 2 : gender: $\operatorname{chi}^{2}(1)=0.08, \mathrm{p}=0.773$; age: $\mathrm{t}(143)=0.53, \mathrm{p}=0.597$; BDI II: $\mathrm{t}(138)=0.68, \mathrm{p}=0.495$; dispersion measure: $Z=-0.06, p=0.950$; translation: $Z=-0.48, p=0.629$; rotation: $Z=-0.34$, $\mathrm{p}=0.734)$.

While all controls completed the fMRI session, three out of 89 patients $(3.5 \%)$ terminated prematurely due to heightened anxiety. Drop-out rates were comparable between groups (Fisher's exact test: $\mathrm{p}=0.121$ ).

Overall mean quality parameters were significantly impaired in patients, showing higher mean percent signal fluctuations, translation and rotation parameters (dispersion measure: $Z=$ $-3.10, p=0.002, d=0.47$; translation: $Z=-2.60, p=0.009, d=0.29$; rotation: $Z=-2.64, p=0.008$, $\mathrm{d}=0.34$; Fig. 1). We observed no differences between female and male subjects in neither group regarding mean data quality (dispersion measure: controls: $Z=-0.54, p=0.589, d=0.10$; patients: $Z=-1.34, p=0.179, d=0.33$; translation: controls: $Z=-0.44, p=0.663, d=0.04$; patients: $Z=-0.79, p=0.430, d=0.27$; rotation: controls: $Z=-0.44, p=0.663, d=0.05$; patients: $\mathrm{Z}=-0.45, \mathrm{p}=0.654, \mathrm{~d}=0.17$ ).

The dispersion measure was comparable for all paradigms in the course of the fMRI session (dispersion measure: controls: $\operatorname{chi}^{2}(2)=0.43, \mathrm{p}=0.808, \mathrm{f}^{2}<0.01$; patients: $\operatorname{chi}^{2}(2)=0.38$, $\left.\mathrm{p}=0.828, \mathrm{f}^{2}=0.15\right)$. We observed a significant time effect for translation and rotation parameters with decreasing values over time in controls, but only a non-significant trend in patients (translation: controls: $\operatorname{chi}^{2}(2)=12.56, \mathrm{p}=0.002, \mathrm{f}^{2}=0.10$; patients: $\operatorname{chi}^{2}(2)=4.97$, $\mathrm{p}=0.084, \mathrm{f}^{2}=0.18$; rotation: controls: $\operatorname{chi}^{2}(2)=15.53, \mathrm{p}<0.001, \mathrm{f}^{2}=0.19$; patients: $\operatorname{chi}^{2}(2)=5.37$, $\left.\mathrm{p}=0.068, \mathrm{f}^{2}=0.10\right)$.

\subsection{Scanner-related distress: differences between patients and controls}

Patients were stronger distressed, reporting significantly higher maximum anxiety, exhaustion and anxiety persistence (maximum anxiety: $\mathrm{t}(129)=8.97, \mathrm{p}<0.0001, \mathrm{~d}=1.87$; exhaustion: $\mathrm{t}$ $(128)=9.92, \mathrm{p}<0.0001, \mathrm{~d}=1.59$; duration: $\mathrm{t}(129)=10.58, \mathrm{p}<0.0001, \mathrm{~d}=1.76)$. The ANOVA on the habituation profile yielded a significant main effect of group (patients $>$ controls; $\mathrm{F}$ ( 1 , $\left.129)=150.06, \mathrm{p}<0.0001, \mathrm{f}^{2}=1.08\right)$, of time course (start-scan $>$ pre-scan $>$ postscan; F (2, $\left.227)=30.56, \mathrm{p}<0.0001, \mathrm{f}^{2}=0.49\right)$ and for the interaction group $*$ time course $(\mathrm{F}(2,227)=5.85$, $\mathrm{p}=0.005, \mathrm{f}^{2}=0.21$ ). Patients exhibited higher distress than controls at all three time points (pre- 
scan, start-scan, post-scan: patients $>$ controls; $p<0.0001)$. Moreover, patients showed significant changes over time, with distress peaking at the start and significant reductions of both anticipatory and scanner-related anxiety towards the end of the session (patients: startscan $>$ pre-scan $>$ post-scan, $p<0.001$ ). In contrast, pre- and post-scan anxiety did not differ in controls (controls: start-scan $>$ pre- scan, $p<0.05$; start-scan $>$ post-scan, $p<0.01$; Fig. 2). No gender effects were observed for subjective distress in patients or controls (controls: maximum anxiety: $\mathrm{t}(52)=1.14, \mathrm{p}=0.258, \mathrm{~d}=0.33$; exhaustion: $\mathrm{t}(52)=0.24, \mathrm{p}=0.809, \mathrm{~d}=0.07$; duration: $\mathrm{t}(52)=0.55, \mathrm{p}=0.586, \mathrm{~d}=0.16$; pre-scan anxiety: $\mathrm{t}(52)=0.14, \mathrm{p}=0.892, \mathrm{~d}=0.04$; startscan anxiety: $\mathrm{t}(52)=0.629, \mathrm{p}=0.53, \mathrm{~d}=0.18$; post-scan anxiety: $\mathrm{t}(52)=-0.49, \mathrm{p}=0.627, \mathrm{~d}=0.14$; patients: maximum anxiety: $\mathrm{t}(74)=-1.14, \mathrm{p}=0.269, \mathrm{~d}=0.27$; exhaustion: $\mathrm{t}(74)=-1.05$, $\mathrm{p}=0.277, \mathrm{~d}=0.26$; duration: $\mathrm{t}(74)=-0.26, \mathrm{p}=0.533, \mathrm{~d}=0.15$; pre-scan anxiety: $\mathrm{t}(74)=-1.52$, $\mathrm{p}=0.134, \mathrm{~d}=0.36$; start-scan anxiety: $\mathrm{t}(74)=-0.34, \mathrm{p}=0.733, \mathrm{~d}=0.08$; post-scan anxiety: $\mathrm{t}$ $(74)=-1.06, \mathrm{p}=0.295, \mathrm{~d}=0.24)$.

\subsection{Identification of vulnerable patients using anxiety measures}

Claustrophobic anxiety was closest related to subjective distress (Table 2), displaying moderate, but significant correlations. Using a median split procedure on the patient group according to CLQ values (Table 3), comparison of low and high CLQ patients showed not only elevated anxiety, but also stronger depressive symptoms in high CLQ patients, so that the BDI II score was used as a covariate in subsequent analyses.

When analyzing differences in subjective distress between high and low claustrophobic patients and healthy controls a main effect for exhaustion, duration and maximum anxiety was observed (exhaustion: $\mathrm{F}(2,124)=38.89, \mathrm{p}<0.0001, \mathrm{f}^{2}=0.79$; duration: $\mathrm{F}(2,123)=37.79$, $\mathrm{p}<0.0001, \mathrm{f}^{2}=0.79$; maximum anxiety: $\left.\mathrm{F}(2,124)=48.18, \mathrm{p}<0.0001, \mathrm{f}^{2}=0.88\right)$. As expected, controls exhibited lower anxiety ratings than both patient groups; high CLQ patients reported significantly stronger exhaustion than low CLQ patients (exhaustion: high CLQ $>$ low CLQ $>$ controls, $\mathrm{p}<0.01$; duration: high CLQ $>$ low CLQ, $\mathrm{p}=0.08$; patients $>$ controls, $\mathrm{p}<0.0001$; maximum anxiety: high CLQ $>$ low CLQ, $p=0.065$, patients $>$ controls, $p<0.0001$ ). During the course of the session differential changes in habituation profiles were observed as indicated by a significant interaction effect of group and time course (main effect group: $\mathrm{F}$ (2, 124) $=67.74, \mathrm{p}<0.0001, \mathrm{f}^{2}=1.05$, high CLQ $>$ low $\mathrm{CLQ}>$ controls, $\mathrm{p}<0.01$; main effect time course: $\mathrm{F}(2,248)=19.91, \mathrm{p}<0.0001, \mathrm{f}^{2}=0.40$, start-scan $>$ pre-scan $>$ post scan, $\mathrm{p}<0.0001$; interaction effect group* time course: $\left.\mathrm{F}(4,248)=4.42, \mathrm{p}=0.003, \mathrm{f}^{2}=0.26\right)$. Controls reported reductions in anxiety from start-scan to post scan only $(\mathrm{p}<0.05)$, while in low CLQ patients anxiety significantly rose immediately during the start of the session (start-scan $>$ pre-scan, post-scan, $\mathrm{p}<0.001$ ). High CLQ patients did not only have a higher level of general anxiety as indicated by the significant main effect of group, but also showed reduced post-scan anxiety levels when compared to pre-scan levels (start-scan $>$ pre-scan $>$ post-scan, $p<0.0001$; see Fig. 2).

High vs. low CLQ subjects also differed in data quality. High, but not low claustrophobic patients showed significantly lower data quality parameters compared to controls, thus limiting this effect to the presence of claustrophobic anxiety to this subgroup (high CLQ vs. controls: dispersion: $\mathrm{Z}=-3.17, \mathrm{p}=0.002, \mathrm{~d}=0.67$; translation: $\mathrm{Z}=-2.73, \mathrm{p}=0.006, \mathrm{~d}=0.31$; rotation: $Z=-2.41, p=0.002, d=0.42$; low $C L Q$ vs. controls: dispersion: $Z=-0.66, p=0.512$, $\mathrm{d}=0.19$; translation: $\mathrm{Z}=-0.31, \mathrm{p}=0.761, \mathrm{~d}=0.05$; rotation: $\mathrm{Z}=-0.81, \mathrm{p}=0.421, \mathrm{~d}=0.09$; high CLQ vs. low CLQ: dispersion: $Z=-2.30, p=0.021, d=0.46$; translation: $Z=-2.04, p=0.042$, $\mathrm{d}=0.40$; rotation: $\mathrm{Z}=-1.28, \mathrm{p}=0.20, \mathrm{~d}=0.35$; see Fig. 1 ). 
Since other anxiety measures, among them anxiety sensitivity, were also related to selfreported distress, the specificity of CLQ results was tested employing the ASI as alternative group divisor within the patient group. Analyses yielded no significant differences between high and low ASI patients (exhaustion: $\mathrm{F}(1,72)=0.92, \mathrm{p}=0.341, \mathrm{f}^{2}=0.12$; duration: $\mathrm{F}(1$, $72)=1.29, \mathrm{p}=0.259, \mathrm{f}^{2}=0.14$; max. anxiety: $\mathrm{F}(1,72)=3.07, \mathrm{p}=0.084, \mathrm{f}^{2}=0.21$; main effect of group: $\mathrm{F}(1,72)=0.55, \mathrm{p}=0.462, \mathrm{f}^{2}=0.09$; main effect of time course: $\mathrm{F}(2,124)=11.41$, $\mathrm{p}<0.0001, \mathrm{f}^{2}=0.40$; interaction effect group* time course: $\left.\mathrm{F}(2,124)=0.22, \mathrm{p}=0.774, \mathrm{f}^{2}=0.06\right)$. Quality parameters were comparable as well (dispersion measure: $Z=-0.38, p=0.70, d=0.06$; translation: $\mathrm{Z}=-0.14, \mathrm{p}=0.882, \mathrm{~d}=0.04$; rotation: $\mathrm{Z}=-0.23, \mathrm{p}=0.816, \mathrm{~d}=0.11$ ).

\subsection{Anxiety management strategies}

Patients told us what had helped or motivated them to go (and remain) in the scanner.

Answers were grouped into seven categories encompassing the following: information ("I was given sufficient information about the scanner and procedure"), experience/exposure ("I want to overcome my anxiety"), research ("I want to support research and help other patients"), company ("someone was there and held my hand"), equipment ("I could not see the scanner through the goggles"), try-out ("I could try-out the scanner before participating in the study"), and cognitive coping strategies ("I knew I could leave the scanner at any time"). Frequencies are displayed in Fig. 3.

\section{Discussion}

The present study is among the first to investigate anxiety-eliciting properties of the MRI scanner itself in a comprehensive sample of patients suffering from PD/AG. The feasibility of large-scale fMRI studies in this vulnerable patient group was tested using drop-out rates and fMRI quality control data as sensitive indicators. Self-reported distress in patients was assessed using a newly developed questionnaire. Special emphasis was laid upon identifying vulnerable patients by anxiety measures. Finally, we aimed to find out how we can make the fMRI session more comfortable for patients.

Results showed that PD/AG patients had no significantly higher drop-out rates. We nevertheless observed significant impairments in data quality, indicating stronger movement artifacts in patients. In line with this, self-reported distress was markedly increased in patients. Higher distress was especially related to claustrophobic anxiety. CLQ, but not ASI scores differentiated between high and low distressed patients. Only high, but not low CLQ patients displayed significantly lower fMRI data quality compared to controls. Patients reported factors helping them to participate in the study, among them provision of information, willingness to self-exposure, interest in research, and cognitive coping strategies.

By successfully scanning $n=86 \mathrm{PD} / \mathrm{AG}$ patients, we were able to prove that comprehensive fMRI studies on PD/AG patients are feasible without significant increases in drop-out rates. Changes in movement parameters over time were observed in controls only, in line with the observation that nervousness (and accompanying movements) may be highest in the beginning of the session. In patients, however, data quality-measures did not change over time. Although mean data quality was lower in patients than controls, effect sizes were small. Subgroup analyses on claustrophobic anxiety moreover indicated, that this effect was restricted to high claustrophobic patients, while low claustrophobic patients were comparable to controls. Regarding realignment parameters, mean translation measures averaged at one $\mathrm{mm}$ which is fairly within limits of tolerable movements. 
Patients were markedly stressed by the scanner environment as indicated by large effect sizes for all group comparisons. We observed pronounced distress levels, being three to four times higher than in controls. Distress also increased in controls when starting the scanner session which is well in line with reports about routine care patients (Melendez and McCrank, 1993). All subjects reported reduced anxiety ratings post scanning, being indicative of a habituation process that appeared to take place in both patients and controls. Only patients, however, showed enhanced pre-scan compared to post-scan values, reflecting elevated anticipatory anxiety.

Claustrophobic anxiety has been reported to predict self-reported distress towards the scanner environment (McGlynn et al., 2003; McIsaac et al., 1998). Although previous studies were conducted on routine care patients or students, we could confirm the relevance of claustrophobic anxiety in this regard for PD/AG patients as well. From all anxiety measures, CLQ scores correlated highest with distress. In line with this, high CLQ, but not high ASI patients showed more self-reported distress and lower data quality, pointing towards a specificity of claustrophobic anxiety for scanner related distress. The prevalence of female patients was twice as high in the high CLQ group. This effect could be interpreted in terms of a higher symptom severity of $\mathrm{PD} / \mathrm{AG}$ especially in female patients. Female and male subjects were however comparable regarding fMRI data quality and subjective distress. Findings on claustrophobic anxiety may indicate that especially those PD/AG patients are at risk for experiencing high levels of distress who have an agoraphobic focus on claustrophobic situations (and not, for example, being in a public, but spaced area or being left alone).

Patients reported several helpful strategies in controlling their anxiety. This is in accordance with previous studies on management strategies for scanner-induced distress (Grey et al., 2000) that employed measures of information delivery (e.g. booklets), counseling, cognitive distraction strategies or relaxation techniques. Since the MRI can act as a powerful anxiety trigger, first attempts using cognitive-behavioral interventions in overcoming scanner related resistance have been conducted (Klonoff et al., 1986). From a therapist's perspective, patients' cognitive avoidance and safety strategies should be eliminated during an exposure session in order to facilitate extinction learning. From a researcher's perspective, however, we would recommend to include as many safety signs into the scanner environment as possible, in order to help the patient to control his/her anxiety.

Physiological processes such as respiration and heart rate may be influenced by different degrees of subjective distress during scanning. Variation of these parameters may account for physiological noise in BOLD data, which may increase error variance and affect the statistical power of fMRI analyses (Birn et al., 2006; Beall, 2010). Due to the absence of physiological coregistrations, we cannot determine if potential alterations in cardio-respiratory processes may have contributed to the higher percentage signal fluctuation seen in high claustrophobic patients.

Scanner distress can furthermore increase neuroendocrine stress markers (Eatough et al., 2009; Tessner et al., 2006). Cortical and subcortical structures that are involved in cognitive and affective processes, including prefrontal structures, hippocampi and amygdala, exert a high density of glucocorticoid-binding receptors (Reul and de Kloet, 1986). Depending on the amount of hormonal reactions, stress responses induced by the MRI setting could in some, but not all, subjects directly modulate neural activity (Muehlhan et al., 2010). As a consequence, interindividual variance in imaging data - either due to different levels of physiological noise or due to direct neurohormonal interactions - may be present. In clinical research, attention 
should therefore be drawn to the comparison of (distressed) patients and (relaxed) healthy controls. With regard to the interpretation of fMRI data it is especially important to carefully control potentially confounding variables, for example by including movement parameters in the fMRI design model on individual subject level and/or exclusion of outliers in the dispersion measure. To reduce the effect of global changes in BOLD response it is especially important to provide effective control conditions in an event related design, assuming that effects of distress are present in both, experimental and control conditions, and are not confounded with the experimental design. However, due to the possible increase of noise or ceiling effects (e.g. within the anxiety network) in the patient data there is a potentially reduced sensitivity, making it especially difficult to interpret an absence of activation in the patient group. Future studies are needed to evaluate the impact of these potentially confounding factors on functional activation patterns (Birn et al., 2009). An alternative approach might be to implement training activities that familiarize subjects with the scanner and thus reduce stress reactivity.

Results should be interpreted within the methodological limits of the study. Being conducted as a multicenter trial, some heterogeneity regarding time/staff resources, possibilities of tryouts and individual handling of patients has to be anticipated. When treating each patient individually in regard to his/her individual needs, standardization of patient interaction may instead be counterproductive. Moreover, different interaction styles may be more representative for MRI centers in general, enhancing external validity of results. All patients included in this study were referred to psychological treatment. This may have influenced motivation for participating in the fMRI study. Low drop-out rates and high compliance may not be representative for other fMRI studies on PD/AG patients. Subjective distress was measured by self-reports only, the study is lacking hormonal or autonomic measures. We can only speculate on the potential influence of self-reported distress on fMRI data via several neurohormonal axes. Although previous studies indicate hormonal responsiveness towards the scanner (Eatough et al., 2009; Tessner et al., 2006), it is yet unknown if results from healthy subjects can be translated to PD/AG patients, where alterations in HPA axis activity have been found (Petrowski et al., 2010). It would be a valuable task for future studies to investigate the influence of the MRI environment on hormonal or autonomic measures in these patients.

In conclusion, the feasibility of large-scale fMRI studies on PD/AG patients that may be especially vulnerable to the stress eliciting properties of the MRI environment could be proved. Studies should stronger acknowledge psychological consequences of the MRI environment itself. Particular attention should be drawn to the adverse effects of the MRI setting that may enhance stress reactions. Future studies are clearly needed to investigate the relationship between self-reported distress and fMRI data in patient groups that are subject to neuroimaging research. 

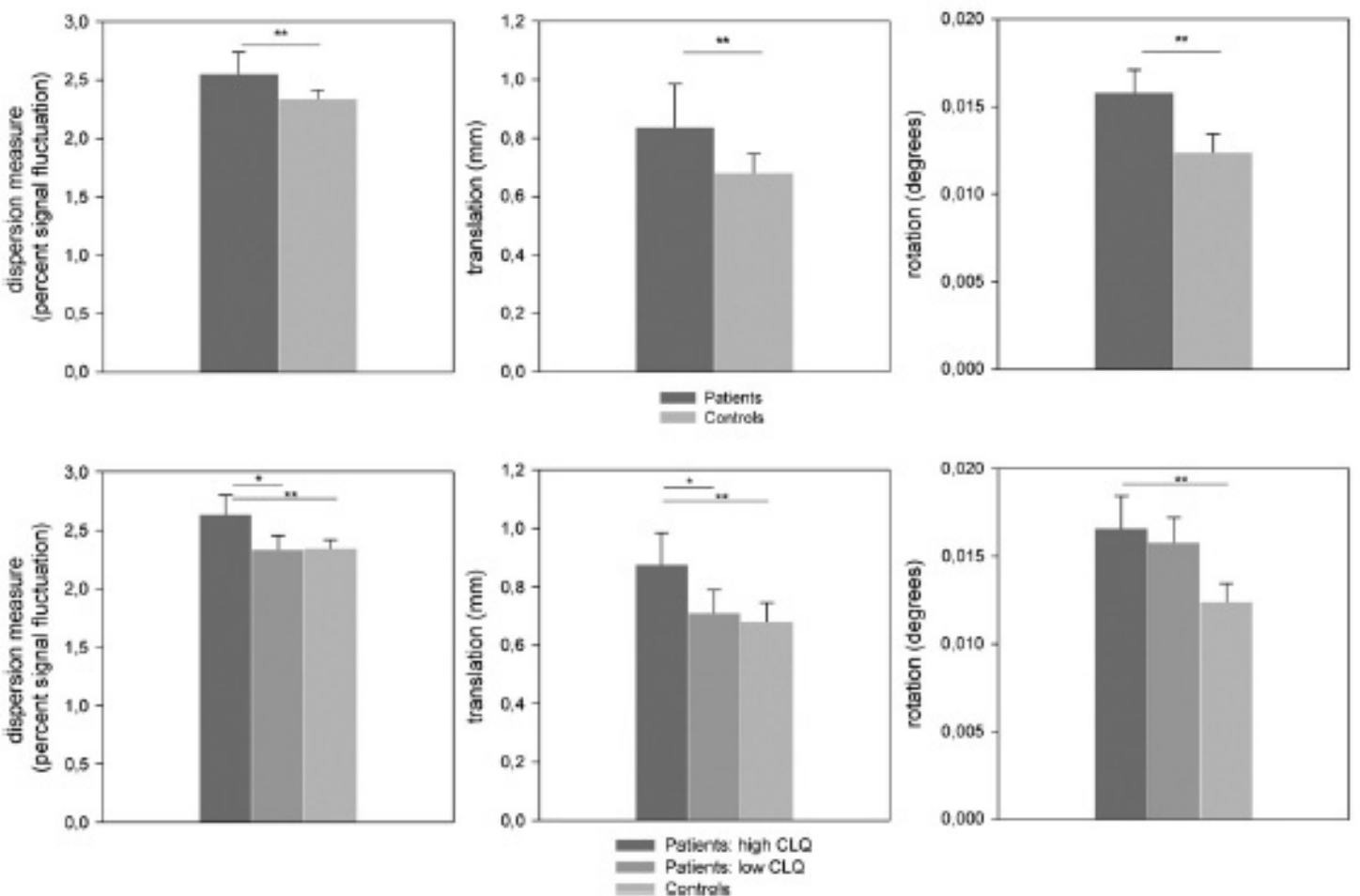

Figure 1 Median fMRI quality parameters (patients and controts: upper half; CQQ median subgroups and controls: bower half; error bars indicate standard error of means). The dispersion measure gives one dispersion measure per volume (averaged across voxets) expressed as percent signal change rela tive to the mean of the time-series. Translation and rotation parameters were derived from realignment procedures using SPM5. CLQ: Claustrophobia Questionnaire. " $p<0.01 ; " p<0.05$.
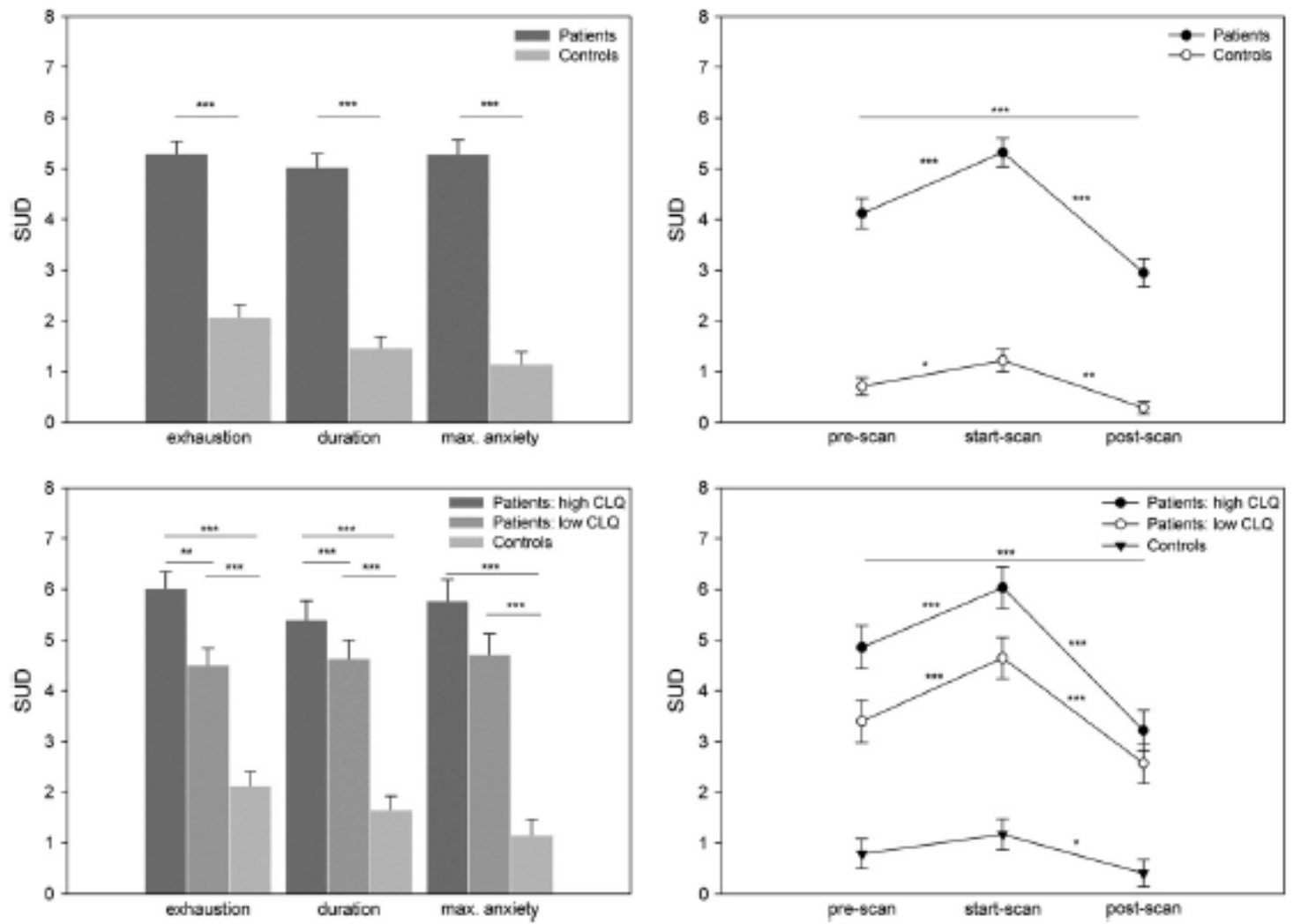

Figure 2 Differences in subjective distress (patients and controls: upper half; CLQ median groups and controls: lower half; error bars indicate standard error of means). SUD: subjective units of distress; QQQ: Claustrophobia Questionnaire. "p <0.05; “"p<0.001; $\ldots$ - $p<0.0001$. 


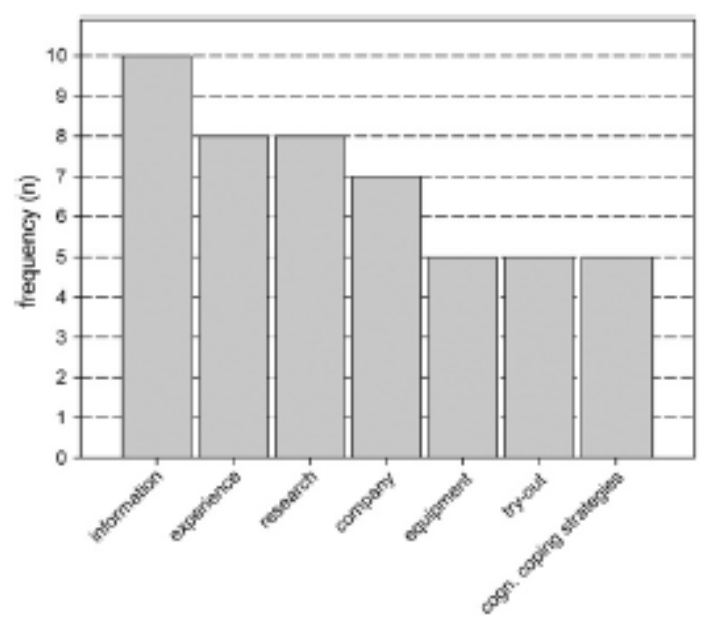

Figure 3 Frequencies of anxiety management strategies (multiple answers possible).

Table 1 Sample characteristics. Means (SD) are given except where noted.

\begin{tabular}{|c|c|c|c|c|c|c|c|c|}
\hline \multirow[b]{3}{*}{ Female gender [n $\left.\left(\xi_{0}\right)\right]$} & \multicolumn{4}{|c|}{ Sample $1(n=179)$} & \multicolumn{4}{|c|}{ Sample 2 ( $n=132)$} \\
\hline & \multicolumn{2}{|c|}{ Patients $(n=89)$} & \multicolumn{2}{|c|}{ Controk $(n=90)$} & \multicolumn{2}{|c|}{ Patients ( $n=77$ ) } & \multicolumn{2}{|c|}{ Controls $(n=55)$} \\
\hline & 61 & $(68.54)$ & 61 & $(67.78)$ & 50 & $(64.94)$ & 36 & $(65.46)$ \\
\hline Age [years] ${ }^{n}$ & 36.48 & (11.31) & 35.56 & (10.87) & 37.25 & $(11.30)$ & 36.56 & (11.49) \\
\hline \multicolumn{9}{|l|}{ Clinical characteristics" } \\
\hline SIGH-A & 24.09 & $(5.21)$ & - & - & 23.75 & $(5.13)$ & - & - \\
\hline PAS & 26.72 & $(9.30)$ & - & - & 25.73 & $(8.92)$ & - & - \\
\hline ASI & 30.26 & $(10.60)$ & - & - & 29.43 & $(10.27)$ & - & - \\
\hline CLQ & 48.09 & $(22.00)$ & $\cdot$ & $\cdot$ & 45.41 & $(21.48)$ & $\cdot$ & $\cdot$ \\
\hline BDI II & 16.49 & $(9.11)$ & 9.51 & $(7.23)$ & 15.82 & $(8.98)$ & 10.36 & $(7.03)$ \\
\hline \multicolumn{9}{|l|}{ FMRI quality parameters } \\
\hline Dispersion measure [percent signal fluctuation] & 3.06 & $(1.72)$ & 2.45 & $(0.71)$ & 2.72 & $(0.90)$ & 2.38 & $(0.61)$ \\
\hline Translation [mm] & 1.19 & (1.37) & 0.88 & $(0.60)$ & 1.00 & $(0.60)$ & 0.90 & $(0.65)$ \\
\hline Rotation [degrees] & 0.02 & $(0.01)$ & 0.02 & $(0.01)$ & 0.02 & $(0.01)$ & 0.02 & $(0.01)$ \\
\hline
\end{tabular}

Sample 1: entire sample; sample 2: sample with fMRI questionnaire; SIGH-A: Hamilton Anxiety Scale; PAS: Panic and Agoraphobia Scale; ASI; Anxiety Sensitivity Index; CLQ: Claustrophobia Questionnaire; BDI II: Beck Depression Inventory.

available for $n=88$ patients (sample 1).

- SIGH-A, PAS, ASI, CLQ available for patients only (sample $1: n-87$ sample $2: n-76$ ), BDIII available for $n=87$ patients/controls (sample 1) and $n=76$ patients $/ n=53$ controls (sample 2 ).

${ }^{c}$ available for $n-88$ patients (sample 1) and $n=76$ patients (sample 2).

Table 2 Association between anxiety measures and subjective distress in patients.

\begin{tabular}{|c|c|c|c|c|c|}
\hline & SIGH-A & PAS & ASI & QQ & BDI ॥ \\
\hline Exhaustion & $0.24^{*}$ & 0.01 & $0.30^{* *}$ & $0.40^{\circ *}$ & $0.28^{\circ}$ \\
\hline Duration & $0.26^{\circ}$ & 0.14 & $0.27^{\circ}$ & $0.27^{\circ}$ & $0.31^{* *}$ \\
\hline Max. anxiety & 0.09 & -0.18 & $0.24^{\circ}$ & $0.23^{\circ}$ & 0.02 \\
\hline \multicolumn{6}{|l|}{ Distress profile } \\
\hline Pre-scan & 0.15 & -0.07 & 0.12 & $0.48^{* *}$ & 0.19 \\
\hline Start-scan & 0.12 & -0.15 & 0.14 & $0.27^{*}$ & -0.03 \\
\hline Post-scan & $0.24^{\circ}$ & 0.01 & $0.30^{* *}$ & $0.40^{\circ *}$ & $0.28^{\circ}$ \\
\hline
\end{tabular}




\begin{tabular}{|c|c|c|c|c|c|c|}
\hline \multirow[b]{2}{*}{ Female gender (n [\%]) } & \multicolumn{2}{|c|}{$\begin{array}{l}\text { Low CLQ patients } \\
(\mathrm{n}=38)\end{array}$} & \multicolumn{2}{|c|}{$\begin{array}{l}\text { High CLQ patients } \\
(n=38)\end{array}$} & \multirow{2}{*}{$\begin{array}{l}\mathrm{Chi}^{2} / \mathrm{T} \text { (df) } \\
12.93 \text { (1) }\end{array}$} & \multirow{2}{*}{$\begin{array}{l}\text { P } \\
<0.0001\end{array}$} \\
\hline & 17 & $(44.7)$ & 32 & $(84.2)$ & & \\
\hline Age (years) & 36.2 & $(10.69)$ & 37.7 & (11.51) & $0.60(74)$ & 0.551 \\
\hline SIGH-A & 22.4 & $(4.24)$ & 25.1 & $(5.63)$ & $-2.33(69)$ & 0.023 \\
\hline PAS $^{a}$ & 25.8 & $(9.42)$ & 25.7 & $(8.52)$ & $0.07(74)$ & 0.945 \\
\hline$A S I^{n}$ & 26.3 & $(9.91)$ & 32.6 & $(9.77)$ & $-2.76(74)$ & 0.007 \\
\hline $\mathrm{CLQ}^{2}$ & 27.3 & $(10.87)$ & 63.5 & (11.99) & $-13.78(74)$ & $<0.0001$ \\
\hline BDI II & 12.8 & $(7.44)$ & 18.8 & $(9.47)$ & $-3.07(70)$ & 0.003 \\
\hline
\end{tabular}

\section{Role of the funding source}

This work is part of the German multicenter trial "Mechanisms of Action in CBT (MAC)". The MAC study is funded by the German Federal Ministry of Education and Research (BMBF; project no. 01GV0615; neuroimaging study: project no. 01GV0611) as part of the BMBF Psychotherapy Research Funding Initiative.

The RTC project was approved by the Ethics Committee of the Medical Faculty of the Technical University of Dresden (EK 164082006). The neuroimaging components were approved by the Ethics Committee of the Medical Faculty of the Rheinisch-Westfaehlische Hochschule University Aachen (EK 073/07). The experimental pharmacology study was approved by the Ethics Committee of the state of Berlin (EudraCT: 2006-00-4860-29). The study was registered with the ISRCTN: ISRCTN80046034.

\section{Contributors}

Author Lueken has contributed to the study design, data collection, managed the literature searches, undertook the statistical analysis and has written the manuscript. Authors Muehlhan, Reinhardt, Wittmann, and Ewert have contributed to the data collection and approved the final manuscript. Author Kellermann has contributed to the data analysis and approved the final manuscript. Authors Wittchen, Konrad, Lang, Ströhle, Gerlach and Kircher have contributed to the study design and approved the final manuscript. Centers

Principal investigators (PI) with respective areas of responsibility in the MAC study are V. Arolt (Münster: Overall MAC Program Coordination), H.U. Wittchen (Dresden: Principal Investigator (PI) for the Randomized Clinical Trial and Manual Development), A. Hamm (Greifswald: PI for Psychophysiology), A.L. Gerlach (Münster: PI for Psychophysiology and Panic subtypes), A. Ströhle (Berlin: PI for Experimental Pharmacology), T. Kircher (Marburg: PI for functional neuroimaging), and J. Deckert (Würzburg: PI for Genetics). Additional site directors in the RTC component of the program are G.W. Alpers (Würzburg), T. Fydrich and L. Fehm (Berlin-Adlershof), and T. Lang (Bremen). Data access and responsibility

All principle investigators take responsibility for the integrity of the respective study data and their components. All authors and coauthors had full access to all study data. Data analysis and manuscript preparation were completed by the authors and coauthors of this article, who take responsibility for its accuracy and content.

\section{Conflict of interest}

The following authors report no conflicts of interest concerning the content of this paper: U. Lueken, M. Muehlhan, H.-U. Wittchen, T. Kellermann, I. Reinhardt, C. Konrad, T. Lang, A. 
Wittmann, A. Gerlach, A. Ewert. A. Ströhle received research funding from the German Federal Ministry of Education and Research, the European Commission (FP6) and Lundbeck, and speaker honoraria from AstraZeneca, Boehringer Ingelheim, Eli Lilly \& Co, Lundbeck, Pfizer, Wyeth and UCB. Educational grants were given by the Stifterverband für die Deutsche Wissenschaft, the Berlin Brandenburgische Akademie der Wissenschaften, the Boehringer Ingelheim Fonds and the Eli Lilly International Foundation. T. Kircher received fees for educational programs from Janssen-Cilag, Eli Lilly, Servier, Lundbeck, Bristol Myers Squibb, Pfizer, and Astra-Zeneca; travel support/sponsorship for congresses from Servier; speaker's honoraria from Janssen-Cilag; and research grants from Pfizer and Lundbeck.

\section{Acknowledgements}

Greifswald (coordinating site for Psychophysiology): Christiane Melzig, Jan Richter, Susan Richter, Matthias von Rad; Berlin-Charité (coordinating Center for Experimental Pharmacology): Harald Bruhn, Anja Siegmund, Meline Stoy, André Wittmann; BerlinAdlershof: Irene Schulz; Münster (Overall MAC Program Coordination, Genetics and Functional Neuroimaging): Andreas Behnken, Katharina Domschke, Adrianna Ewert, Carsten Konrad, Bettina Pfleiderer, Peter Zwanzger; Münster (coordinating site for psychophysiology and subtyping): Judith Eidecker, Swantje Koller, Fred Rist, Anna Vossbeck-Elsebusch; Marburg/Aachen (coordinating center for functional neuroimaging): Barbara Drüke, Sonja Eskens, Thomas Forkmann, Siegfried Gauggel, Susan Gruber, Andreas Jansen, Thilo Kellermann, Isabelle Reinhardt, Nina Vercamer-Fabri; Dresden (coordinating site for data collection, analysis, and the RCT): Franziska Einsle, Christine Froehlich, Andrew T. Gloster, Christina Hauke, Simone Heinze, Michael Hoefler, Ulrike Lueken, Peter Neudeck, Stephanie Preiß, Dorte Westphal; Würzburg Psychiatry Department (coordinating center for genetics): Andreas Reif; Würzburg Psychology Department: Julia Duerner, Hedwig Eisenbarth, Antje B. M. Gerdes, Harald Krebs, Paul Pauli, Silvia Schad, Nina Steinhäuser; Bremen: Veronika Bamann, Sylvia Helbig-Lang, Anne Kordt, Pia Ley, Franz Petermann, Eva-Maria Schroeder. Additional support was provided by the coordinating center for clinical studies in Dresden (KKS Dresden): Xina Graehlert and Marko Käppler.

\section{References}

- Arolt, V., Zwanziger, P., Strohle, A., Hamm, A., Gerlach, A., Kircher, T., Deckert, J., 2009. The research network PANIC-NET: improving the treatment of panic disorder from a better understanding of fear circuit mechanisms to more effective psychological treatment and routine care. Psychother. Psychosom. Med. Psychol. 59, 124-131.

- Bandelow, B., 1999. Panic and Agoraphobia Scale (PAS). Ashland, Hogrefe \& Huber Publishers.

- Beall, E.B., 2010. Adaptive cyclic physiological noise modelling and correction in functional MRI. J. Neurosci. Methods 187, 216-228.

- Beck, A.T., Steer, R.A., Brown, G.K., 1996. Beck Depression Inventory, 2nd ed. The Psychological Corporation, San Antonio.

- Birn, R.M., Diamond, J.B., Smith, M.A., Bandettini, P.A., 2006. Separating respiratory-variation-related neuronal-activity-related fluctuations in fluctuations from fMRI. Neuroimage 31,1536-1548.

- $\quad$ Birn, R.M., Murphy, K., Handwerker, D.A., Bandettini, P.A., 2009. fMRI in the presence of task-correlated breathing variations. Neuroimage 47, 1092-1104. 
- Bystritsky, A., Pontillo, D., Powers, M., Sabb, F.W., Craske, M.G., Bookheimer, S.Y., 2001. Functional MRI changes during panic anticipation and imagery exposure. NeuroReport 12, 3953-3957.

- Cohen, J., 1983. Statistical Power Analysis for the Behavioral Sciences. Lawrence Erlbaum, Hillsdale.

- Dantendorfer, K., Amering, M., Bankier, A., Helbich, T., Prayer, D., Youssefzadeh, S., Alexandrowicz, R., Imhof, H., Katschnig, H., 1997. A study of the effects of patient anxiety, perceptions and equipment on motion artifacts in magnetic resonance imaging. Magn. Reson. Imaging 15, 301-306.

- Eatough, E.M., Shirtcliff, E.A., Hanson, J.L., Pollak, S.D., 2009. Hormonal reactivity to MRI scanning in adolescents. Psychoneuroendocrinology 34, 1242-1246.

- Fishbain, D.A., Goldberg, M., Labbe, E., Zacher, D., Steelerosomoff, R., Rosomoff, H., 1988. Long-term claustrophobia following magnetic-resonance imaging. Am. J. Psychiatry 145, 1038-1039.

- Flaherty, J.A., Hoskinson, K., 1989. Emotional distress during magnetic resonance imaging. N. Engl. J. Med. 320, 467-468 16-2.

- Gloster, A.T., Wittchen, H.U., Einsle, F., Hofler, M., Lang, T., Helbig-Lang, S., Fydrich, T., Fehm, L., Hamm, A.O., Richter, J., Alpers, G.W., Gerlach, A.L., Strohle, A., Kircher, T., Deckert, J., Zwanzger, P., Arolt, V., 2009. Mechanism of action in CBT (MAC): methods of a multi-center randomized controlled trial in 369 patients with panic disorder and agoraphobia. Eur. Arch. Psychiatry Clin. Neurosci. 259 (Suppl 2), S155-S166.

- Grey, S.J., Price, G., Mathews, A., 2000. Reduction of anxiety during MR imaging: a controlled trial. Magn. Reson. Imaging 18, 351-355.

- Guy, W., 1976. ECDEU assessment manual for psychopharmacology. Rev. Rockville, MD, U.S. National Institute of Health, Psychopharmacology Research Branch.

- Kilborn, L.C., Labbe, E.E., 1990. Magnetic-resonance-imaging scanning procedures development of phobic response during scan and at one-month follow-up. J. Behav. Med. 13, 391-401.

- Klonoff, E.A., Janata, J.W., Kaufman, B., 1986. The use of systematic-desensitization to overcome resistance to Magnetic-Resonance-Imaging (MRI) scanning. J. Behav. Ther. Exp. Psychiatry 17, 189-192.

- Mackenzie, R., Sims, C., Owens, R.G., Dixon, A.K., 1995. Patients perceptions of magnetic-resonance-imaging. Clin. Radiol. 50, 137-143.

- Maddock, R.J., Buonocore, M.H., Kile, S.J., Garrett, A.S., 2003. Brain regions showing increased activation by threat-related words in panic disorder. NeuroReport $14,325-328$.

- McGlynn, F.D., Karg, R., Lawyer, S.R., 2003. Fear responses to mock magnetic resonance imaging among college students: toward a prototype experiment. J. Anxiety Disord. 17, 335-347.

- McIsaac, H.K., Thordarson, D.S., Shafran, R., Rachman, S., Poole, G., 1998. Claustrophobia and the magnetic resonance imaging procedure. J. Behav. Med. 21, 255-268.

- Melendez, J.C., McCrank, E., 1993. Anxiety-related reactions associated with magnetic-resonance-imaging examinations. JAMA-J. Am. Med. Assoc. 270, 745-747.

- Muehlhan, M., Lueken, U., Wittchen, H.-U., Kirschbaum, C., 2010. The scanner as a stressor: Evidence from subjective and neuroendocrine stress parameters in the time course of a functional magnetic resonance imaging session. Int. J. Psychophysiol. doi:10.1016/j.ijpsycho.2010.09.009. 
- Paulus, M.P., 2008. The role of neuroimaging for the diagnosis and treatment of anxiety disorders. Depress. Anxiety 25, 348-356.

- Petrowski, K., Herold, U., Joraschky, P., Wittchen, H.U., Kirschbaum, C., 2010. A striking pattern of cortisol non-responsiveness to psychosocial stress in patients with panic disorder with concurrent normal cortisol awakening responses. Psychoneuroendocrinology 35, 414-421.

- $\quad$ Pillay, S.S., Gruber, S.A., Rogowska, J., Simpson, N., Yurgelun-Todd, D.A., 2006. fMRI of fearful facial affect recognition in panic disorder: the cingulate gyrusamygdala connection. J. Affect. Disord. 94, 173-181.

- Radomsky, A.S., Rachman, S., Thordarson, D.S., McIsaac, H.K., Teachman, B.A., 2001. The claustrophobia questionnaires. J. Anxiety Disord. 15, 287-297.

- Reiss, S., Peterson, R.A., Gursky, D.M., McNally, R.J., 1986. Anxiety sensitivity, anxiety frequency and the prediction of fearfulness. Behav. Res. Ther. 24, 1-8.

- Reul, J.M., de Kloet, E.R., 1986. Anatomical resolution of two types of corticosterone receptor sites in rat brain with in vitro autoradiography and computerized image analysis. J. Steroid Biochem. 24, 269-272.

- Shear, M.K., Vander, B.J., Rucci, P., Endicott, J., Lydiard, B., Otto, M.W., Pollack, M.H., Chandler, L., Williams, J., Ali, A., Frank, D.M., 2001. Reliability and validity of a structured interview guide for the Hamilton Anxiety Rating Scale (SIGHA). Depress. Anxiety 13, 166-178.

- Spiegelhalder, K., Hornyak, M., Kyle, S.D., Paul, D., Blechert, J., Seifritz, E., Hennig, J., van Elst, L.T., Riemann, D., Feige, B., 2009. Cerebral correlates of heart rate variations during a spontaneous panic attack in the fMRI scanner. Neurocase 15, 527534.

- Stocker, T., Schneider, F., Klein, M., Habel, U., Kellermann, T., Zilles, K., Shah, N.J., 2005. Automated quality assurance routines for fMRI data applied to a multicenter study. Hum. Brain Mapp. 25, 237-246.

- Tessner, K.D., Walker, E.F., Hochman, K., Hamann, S., 2006. Cortisol responses of healthy volunteers undergoing magnetic resonance imaging. Hum. Brain Mapp. 27, 889-895.

- Wittchen, H.-U., Pfister, H., 1997. DIA-X Interview. Frankfurt, Swets \& Zeitlinger. 